\title{
Noninvasive Investigation of the Presence and Extent of Coronary Artery Disease by the Evaluation of Fingertip-Reactive Hyperemia
}

\author{
Sefik Gorkem Fatihoglu ${ }^{a}$ Farzin Jam ${ }^{b}$ Sercan Okutucu ${ }^{c} \quad$ Ali Oto ${ }^{c}$

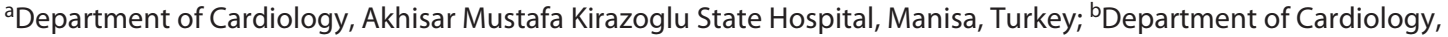 \\ Akademi Hospital, Izmit, Turkey; 'Department of Cardiology, Memorial Ankara Hospital, Ankara, Turkey
}

\section{Highlights of the Study}

- Classical risk factors are insufficient to explain the pathophysiology of coronary artery disease. Deterioration of endothelial functions has a role in the development of atherosclerotic plaques.

- The measurement of reactive hyperemia index by fingertip-reactive hyperemia-peripheral artery tonometry in low-risk to medium-risk individuals in clinical practice may help in clinical decision-making processes.

\section{Keywords}

Coronary artery disease $\cdot$ Endothelial dysfunction $\cdot$ Reactive hyperemia index · Peripheral arterial tonometry

\footnotetext{
Abstract

Objective: Fingertip-reactive hyperemia-peripheral artery tonometry (RH-PAT) is an emerging novel noninvasive method for evaluating endothelial function. We aimed to evaluate endothelial function with fingertip-reactive hyperemia by RH-PAT in symptomatic patients undergoing elective coronary angiography and to assess the relationship between the degree of endothelial dysfunction (ED) and the presence and the severity of coronary artery disease (CAD). Subjects and Methods: We assessed 92 patients. Before coronary angiography, endothelial function was measured by RH-PAT and reactive hyperemia index $(\mathrm{RHI})$ was obtained. For each patient, the Gensini score was calculated according to the
}

coronary angiographic findings to evaluate the severity of CAD. Results: In CAD (+) group, RHI (1.35 \pm 0.57 vs. $1.74 \pm$ $0.46, p=0.001$ ) mean values were lower than CAD (-) group. In multivariate logistic regression analysis, age, high-density lipoprotein, and $\mathrm{RHI}$ were found to be independent parameters predicting the presence of CAD. In multivariate linear regression analysis, $\mathrm{RHI}$ was found to be an independent predictor associated with the severity of CAD. In receiver operating characteristic curve analysis, $\mathrm{RHI}$ achieved an area under the curve of 0.763 (95\% confidence interval $0.663-$ $0.846, p=0.001)$ for the ability to predict the presence of CAD. A cut-off value of 1.49 for an $\mathrm{RHI}$ predicted the presence of CAD with a sensitivity of $75 \%$ and a specificity of $72.9 \%$. Conclusion: $\mathrm{RH}-\mathrm{PAT}$ is an objective and highly reproducible test with superior diagnostic accuracy for the evaluation of ED. We found significant correlations between ED and the presence and the severity of CAD. @ 2022 The Author(s).

Published by S. Karger AG, Basel
C 2022 The Author(s).

Published by S. Karger AG, Basel

This is an Open Access article licensed under the Creative Commons Attribution-NonCommercial-4.0 International License (CC BY-NC) (http://www.karger.com/Services/OpenAccessLicense), applicable to the online version of the article only. Usage and distribution for commercial purposes requires written permission. 


\section{Introduction}

Despite the development of diagnostic and therapeutic advancements and treatment methods, cardiovascular diseases remain the leading cause of mortality worldwide. Various significant risk factors have been defined for the development of coronary artery disease (CAD) [1]. However, classical risk factors are absent in one-third of all CAD cases, and the lipid levels are normal in half of the cases. Therefore, classical risk factor control is often insufficient in explaining the pathophysiology of CAD [2, 3]. Endothelial dysfunction (ED) is currently among the most investigated novel risk factors.

Studies from the last two decades have shown that the deterioration of endothelial functions has a role in the development of, and complications associated with, atherosclerotic plaques. ED is characterized by the reduction of the bioavailability of vasodilators, particularly nitric oxide (NO), and/or an increase in endothelium-derived contracting factors. The resulting imbalance leads to an impairment of endothelium-dependent vasodilation, which is the functional characteristic of ED. In addition to the impaired endothelium-dependent vasodilation, ED also consists of a specific state of endothelial activation, which is characterized by pro-inflammatory, proliferative, and procoagulatory states that favor all stages of atherosclerosis [4-6].

Fingertip-reactive hyperemia-peripheral artery tonometry (RH-PAT) is an emerging novel noninvasive method for evaluating endothelial function. Fingertip $\mathrm{NO}$ and pulse amplitude increase as a result of reactive hyperemia. Clinical trials have shown that the decreased pulse amplitude response to hyperemia is associated with ED in the coronary artery $[7,8]$. This study aimed to evaluate fingertip RH-PAT in patients with suspected CAD and to investigate the relationship between RH-PAT and the presence and prevalence of CAD.

\section{Subjects and Methods}

The study included a total of 92 patients ( 33 females and 59 males) who were scheduled for elective coronary angiography due to cardiac symptoms, ECG changes, or positive stress tests. The exclusion criteria were as follows: acute coronary syndrome, known CAD, heart failure, significant heart valve disease, patients with nonsinus rhythm in ECG, alcohol use, vitamin use, active infectious and autoimmune diseases, neoplastic diseases, and chronic liver and kidney diseases.

Peripheral artery tonometry was performed using the EndoPAT 2000 (Itamar Medical Ltd., Caesarea, Israel). Peripheral artery tonometry was performed for all patients 1 day before coro- nary angiography and at least $1 \mathrm{~h}$ after flow mediated dilatation (FMD) measurement. Patients were asked to fast and not smoke or consume alcohol, caffeine, vitamins, or medication for at least $12 \mathrm{~h}$ prior to the measurement which was made in a quiet setting at $21^{\circ} \mathrm{C}-23^{\circ} \mathrm{C}$. The patients' blood pressure and heart rate were measured $5 \mathrm{~min}$ before the procedure. Patients were seated in a comfortable position in front of the table on which the EndoPAT 2000 device was placed. The computer software was started (Endo-PAT 2000 version 3.2.4; Itamar Medical Ltd.), and the patient's first name, last name, age, weight, height, and systolic and diastolic blood pressures were recorded. Both index fingers were properly placed into the probes and the cables were anchored to the patient's arm. Air was removed from inside the probe by pressing the deflate button and the probes were subsequently made to grasp the finger through air pressure by pressing the inflate button. Basal recordings were then made for 5 min by pressing the record button from the computer software. The sphygmomanometer cuff was then placed on one arm. It was inflated to an average pressure of $200 \mathrm{~mm} \mathrm{Hg}$ and recordings were made in this condition for $5 \mathrm{~min}$, and then the cuff was quickly removed, and a 5-min postocclusion recording was made [9]. The recordings made by the computer software were automatically analyzed by the software independently of the technician and the results were recorded as reactive hyperemia index (RHI) (Fig. 1).

All subjects underwent selective coronary angiography through the femoral artery using the Judkins technique under local anesthesia (Coroscop, Siemens, Munich, Germany). All coronary angiograms were evaluated by 2 experienced cardiologists who were blinded to the laboratory results of the subjects. The presence and the prevalence of CAD were determined according to the Gensini scores $[10,11]$. A Gensini score greater than zero indicated a presence of CAD.

\section{Statistical Analysis}

Distribution of data was assessed by using a one-sample Kolmogorov-Smirnov test. Data are demonstrated as mean \pm SD for normally distributed continuous variables, median (minimum to maximum) for skew-distributed continuous variables, and frequencies for categorical variables. An independent-sample $t$ test and the Mann-Whitney $U$ test for numerical variables and Pearson's $\chi^{2}$ test or Fisher's exact test for categorical variables were used for intergroup comparisons. Statistical analysis of the data was conducted using Statistical Package for Social Sciences (SPSS) for Windows 20 (IBM SPSS Inc., Chicago, IL, USA) ve MedCalc 11.4.2 (MedCalc Software, Mariakerke, Belgium) $p<0.05$ was considered statistically significant. Univariate logistic regression analysis was used to analyze the effect of parameters like age, gender, diabetes, hypertension, total cholesterol, low-density lipoprotein, high-density lipoprotein levels, body mass index, waist circumference, and RHI on CAD and the risk factors that found to be significant were added to multivariate logistic regression model. Univariate linear regression analysis was made to assess the effect of the same parameters on Gensini score and the parameters that found to be significant were used in multivariate linear regression analysis. Pearson and Spearmen correlation analysis was used to assess the relationship of variables (like Gensini score and RHI) between each other. The cut-off value of RHI for predicting $\mathrm{CAD}$ was investigated with the receiver operating characteristic curve. 


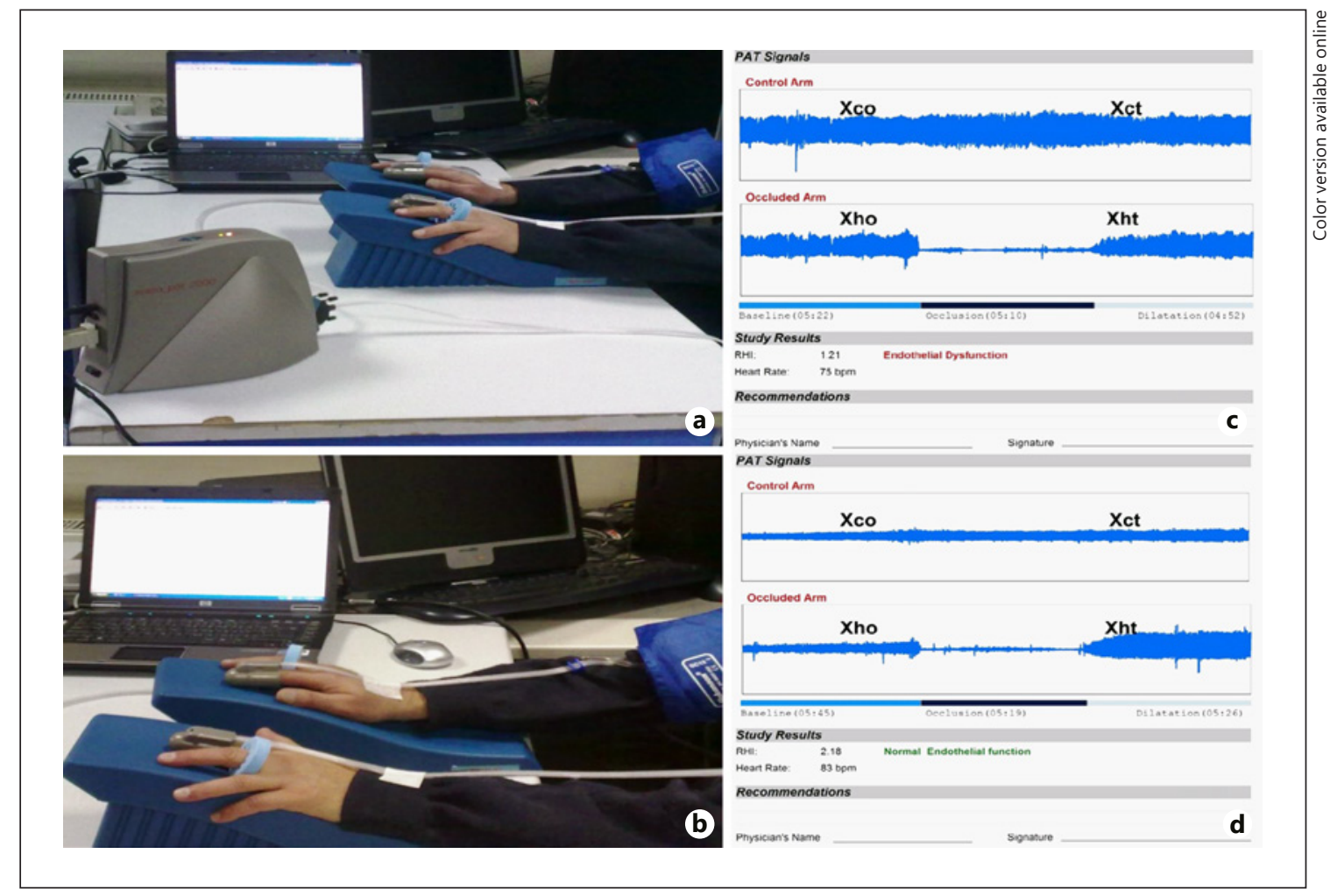

Fig. 1. Measurement of reactive hyperemia-peripheral arterial tonometry, Endo-PAT 2000 device (a) system and finger alignment (b) are shown. On the right side, an example of an HF patient with ED (c) and normal endothelial function (d) are shown.

Table 1. Demographic characteristics, associated risk factors, medications, and laboratory findings

\begin{tabular}{ll}
\hline Parameter & Total $(n=92)$ \\
\hline Age, year & $59.7 \pm 12.0$ \\
Gender, male, $n(\%)$ & $59(64.1)$ \\
BMl, kg/m² & $29.2 \pm 4.4$ \\
Waist circumference, cm & $102.4 \pm 12.4$ \\
Diabetes, $n$ (\%) & $27(29.3)$ \\
Hypertension, $n(\%)$ & $48(52.2)$ \\
Hyperlipidemia, $n(\%)$ & $28(30.4)$ \\
Smokers, $n$ (\%) & $22(23.9)$ \\
Acetylsalicylic acid, $n(\%)$ & $31(33.7)$ \\
Beta blocker, $n$ (\%) & $19(20.7)$ \\
Calcium channel blocker, $n(\%)$ & $10(10.8)$ \\
ACE inhibitor, $n$ (\%) & $35(38.0)$ \\
Statin, $n(\%)$ & $25(27.2)$ \\
LDL, mg/dL & $132.9 \pm 34.6$ \\
HDL, mg/dL & $50.4 \pm 13.8$ \\
Triglyceride, mg/dL & $161.0 \pm 84.2$ \\
Fasting blood glucose, mg/dL & $107.3 \pm 37.4$ \\
Mean RHI & $1.55 \pm 0.42$ \\
\hline
\end{tabular}

BMI, body mass index; LDL, low-density lipoprotein; HDL, highdensity lipoprotein; $\mathrm{RHI}$, reactive hyperemia index.

\section{Results}

Demographic characteristics, associated risk factors, medications, and laboratory findings of the 92 subjects (33 females, 59 males; mean age $59.7 \pm 12.0$ years) are presented in Table 1.

Demographic characteristics, risk factors, medications, and laboratory findings of the groups according to the presence of CAD as detected by coronary angiography are shown in Table 2. Mean RHI was lower in patients with CAD ( $1.35 \pm 0.57$ vs. $1.74 \pm 0.46, p=0.001)$.

In this study, all parameters that were thought to be associated with $\mathrm{CAD}$ were analyzed using univariate logistic regression. RHI was found to be an independent predictor of $\mathrm{CAD}(\mathrm{OR}=0.035,95 \% \mathrm{CI}=0.006-0.195, p=0.001)$. All parameters that were determined to be significantly associated with CAD in univariate logistic regression analysis were collectively analyzed with multivariate logistic regression analysis (Table 3 ). After the analysis of the parameters associated with CAD, it was found that RHI was an independent predictor of the presence of CAD (OR $=0.069,95 \% \mathrm{CI}=0.070-0.689, p=0.023)$. The indepen- 
Table 2. Demographic characteristics, risk factors, drugs, and laboratory findings of the groups according to the presence of CAD as detected by coronary angiography

\begin{tabular}{llll}
\hline & CAD $(+)(n=44)$ & CAD $(-)(n=48)$ & $p$ value \\
\hline Age, years & $64.4 \pm 10.5$ & $55.2 \pm 11.7$ & 0.001 \\
BMI, kg/m ${ }^{2}$ & $29.0 \pm 4.7$ & $29.40 \pm 4.03$ & 0.694 \\
Waist circumference, cm & $104.2 \pm 12.8$ & $100.6 \pm 11.8$ & 0.170 \\
Diabetes, $n$ (\%) & $17(38.6)$ & $10(20.8)$ & 0.061 \\
Hypertension, $n$ (\%) & $29(65.9)$ & $19(39.6)$ & 0.012 \\
Hyperlipidemia, $n$ (\%) & $15(34.1)$ & $13(27.1)$ & 0.153 \\
Smokers, $n$ (\%) & $10(22.7)$ & $12(25.0)$ & 0.031 \\
Aspirin, $n(\%)$ & $21(47.7)$ & $10(20.8)$ & 0.006 \\
Beta blocker, $n(\%)$ & $11(25.0)$ & $8(16.7)$ & 0.324 \\
Calcium channel blocker, $n(\%)$ & $7(15.9)$ & $3(6.3)$ & 0.137 \\
ACE inhibitor, $n$ (\%) & $21(47.7)$ & $14(29.2)$ & 0.067 \\
Statin, $n$ (\%) & $15(48.4)$ & $10(20.8)$ & 0.153 \\
LDL, mg/dL & $132.8 \pm 28.9$ & $133.2 \pm 39.6$ & 0.959 \\
HDL, mg/dL & $46.9 \pm 11.8$ & $53.6 \pm 14.9$ & 0.020 \\
Triglycerides, mg/dL & $176.5 \pm 96.6$ & $146.9 \pm 69.2$ & 0.093 \\
Fasting glucose, mg/dL & $116.8 \pm 45.3$ & $98.8 \pm 26.1$ & 0.024 \\
Mean RHI & $1.35 \pm 0.57$ & $1.74 \pm 0.46$ & 0.001 \\
\hline
\end{tabular}

BMI, body mass index; LDL, low-density lipoprotein; HDL, high-density lipoprotein; CAD, coronary artery disease; $\mathrm{RHI}$, reactive hyperemia index.

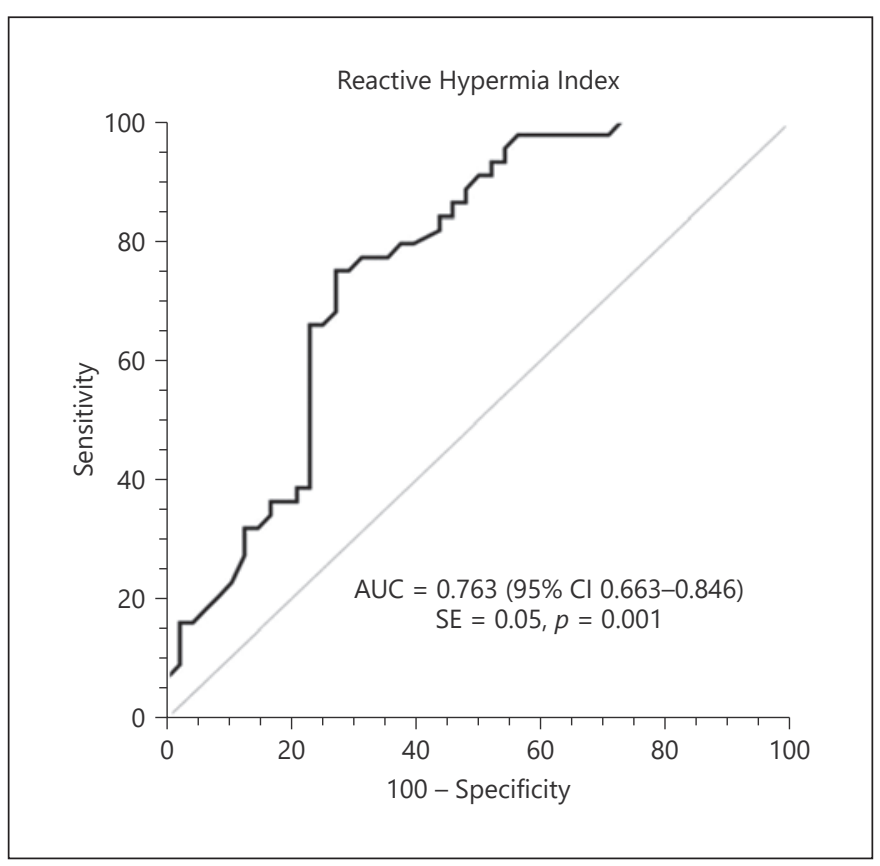

Fig. 2. ROC analysis for RHI in predicting CAD risk.

dent variables of age, high-density lipoprotein, and RHI were determined to collectively predict CAD by $56.6 \%$ (Nagelkerke's $R^{2}=0.056$ ). In addition, the model created with these variables correctly estimated the outcome of 33 of the $44 \mathrm{CAD}$-positive subjects and 41 of the $48 \mathrm{CAD}$ -
Table 3. Multivariate logistic regression analysis of parameters that were determined to be significantly associated with CAD in univariate logistic regression analysis

\begin{tabular}{lllll}
\hline & Wald $x^{2}$ & $p$ value & Odds ratio & $95 \% \mathrm{Cl}$ \\
\hline Year & 8.102 & 0.004 & 1.097 & $1.029-1.169$ \\
Smoking & 2.296 & 0.130 & 2.905 & $0.731-11.536$ \\
HDL & 4.378 & 0.036 & 0.944 & $0.894-0.996$ \\
Creatinine & 0.711 & 0.399 & 0.220 & $0.07-7.424$ \\
DBP & 3.022 & 0.082 & 1.060 & $0.993-1.132$ \\
RHI & 5.188 & 0.023 & 0.069 & $0.07-0.689$ \\
\hline
\end{tabular}

DBP, diastolic blood pressure; HDL, high-density lipoprotein, $\mathrm{RHI}$, reactive hyperemia index.

negative subjects. Hence, our model of 3 variables correctly predicted the outcome of $80.4 \%$ of all subjects.

All parameters that were thought to impact the severity of CAD, i.e., the Gensini score, were analyzed using univariate linear regression analysis. The Gensini score was used to quantify the severity of CAD. RHI was found to be an independent negative predictor of CAD severity $(\beta+\mathrm{SE}=24.079 \pm 5.660, p=0.001)$. Using multivariate linear regression analysis, RHI was determined to be an independent variable for the Gensini score $(\beta+\mathrm{SE}=$ $-13.483 \pm 5.440, p=0.015$ ).

Based on the abovementioned results, we conclude that RHI is a parameter capable of predicting both the 
Fig. 3. Association of RHI with the Gensini score.

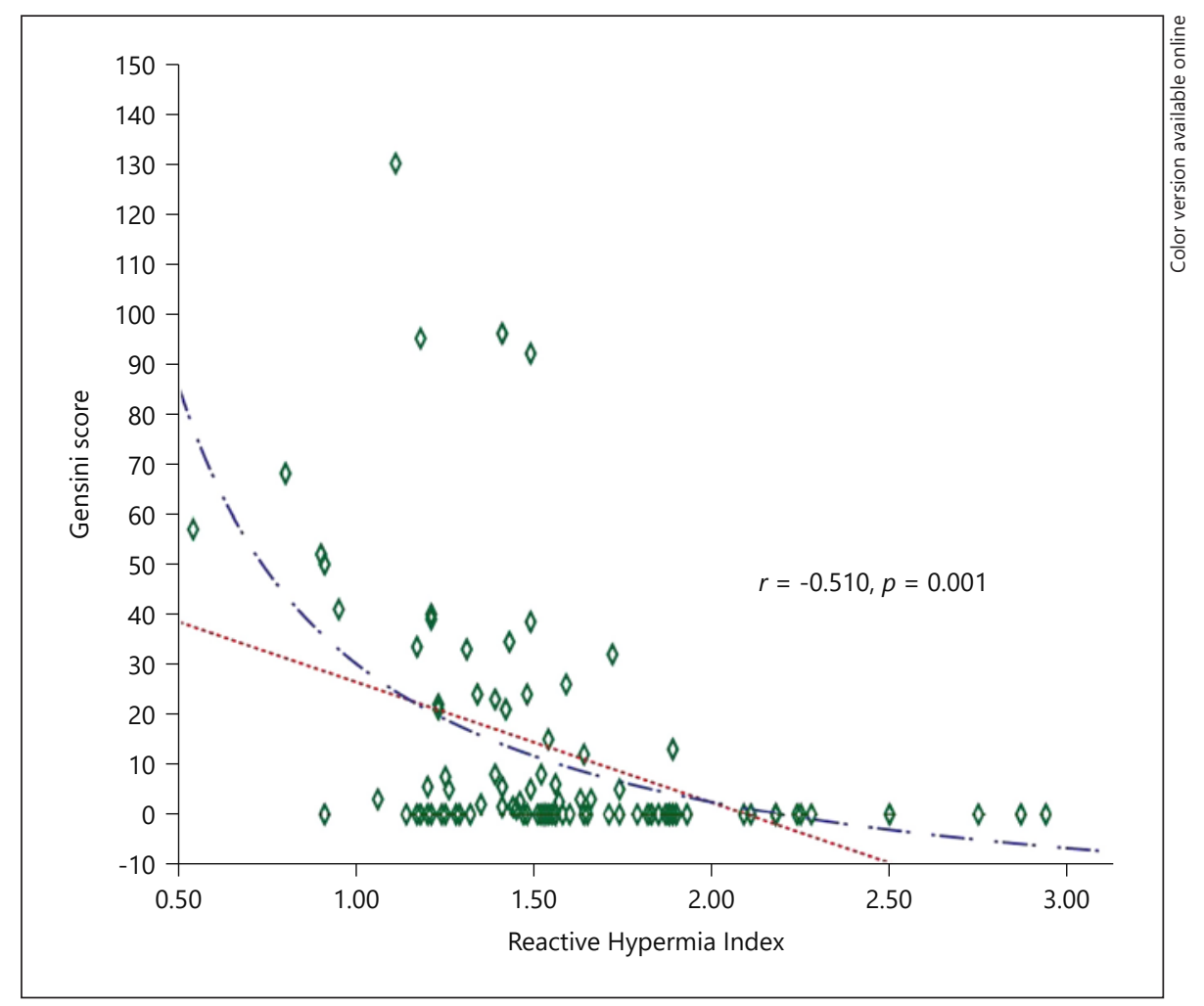

presence and the severity of CAD. The cut-off value of RHI, identified as an independent risk factor for CAD, was investigated with the receiver operating characteristic curve and the area under the curve was found to be 0.763 (95\% CI $=0.663-0.846, p=0.001$ ) (Fig. 2). The RHI cut-off value of 1.49 was found to predict the presence of CAD with $75 \%$ sensitivity and $72.9 \%$ specificity. The RHI and the Gensini score were moderately negatively correlated, and this correlation was statistically significant $(r=$ $-0.510, p=0.001)$. The Gensini score decreased with increasing RHI (Fig. 3).

\section{Discussion}

The main finding of the present study is that RHI values are lower in CAD (+) group than CAD (-) group. In symptomatic patients, assessment of endothelial function with peripheral artery tonometer predicts the presence and severity of CAD. An RHI cut-off value of 1.49 predicts the presence of CAD with a sensitivity of $75 \%$ and specificity of $72.9 \%$.

$\mathrm{ED}$ is known to play a role in the onset of atherosclerosis. The presence of ED in the coronary arteries of advanced atherosclerosis patients was first demonstrated in
1986 [12]. Abnormal endothelial physiology plays a role both in the early stage progression and in the late stages of atherosclerosis and the control of dynamic coronary plaques. Several studies have investigated this close relationship between ED and atherosclerosis. Many of these studies used not only invasive or noninvasive methods but also non-reproducible methods to measure endothelial functions.

Kuvin et al. [13] divided 60 patients with ischemia findings in myocardial perfusion scintigraphy or $>50 \%$ stenosis in coronary angiography into groups according to the presence of CAD. They assessed FMD with brachial artery ultrasonography and RHI with the EndoPAT 2000. They found both FMD and RHI to be lower in the CAD group (RHI: $2.0 \pm 0.1$ vs. $2.4 \pm 0.1, p=0.001$ ). Similarly, we found that ED was greater in the group with CAD and that FMD and RHI were lower in the CADpositive group. In our study, RHI was $1.35 \pm 0.57$ for the CAD-positive group and $1.74 \pm 0.46$ for the CAD-negative group. The difference between our results and those of the aforementioned study may have resulted from the methods used for the diagnosis of CAD [13].

Data on the correlation of ED and the prevalence of CAD are scarce. Both the measurement of endothelial functions by PAT, a sensitive and highly reproducible 
method, and the measurement of CAD by coronary angiography, a gold-standard method, have facilitated the investigation of the relationship between $\mathrm{ED}$ and CAD. Thus, the obtained results are comparatively reliable and less open-ended.

Suwaidi et al. [14] administered intracoronary acetylcholine infusion to 157 patients with mild CAD $(<40 \%$ stenosis in coronary angiography) to evaluate endothelial function and measured the subjects' responses to divide them into 3 groups: mild, moderate, and severe ED. At the end of 2 years of follow-up, none of the subjects in the mild or moderate ED groups developed major cardiovascular events, whereas the incidence of major cardiovascular events was $14 \%$ in the severe ED group. In a similar study, Schachinger et al. [15] followed 147 patients for about 8 years and found that major cardiovascular events were more likely in subjects with impaired endothelial response to intracoronary acetylcholine. In this study, ED was shown as an independent risk factor for predicting adverse events in patients with CAD. Rubinshtein et al. [16] evaluated 270 patients with no significant lesions in their coronary arteries for ED with the Endo-PAT 2000 and followed their subjects for 7 years. They found that major cardiovascular events were more likely in patients with low RHI.

In light of the studies mentioned above, there is sufficient evidence to indicate a relationship between ED and atherosclerosis [17]. The relationship between these two processes can be explained by several different mechanisms. ED is accompanied by decreased vasodilators, the most significant being decreased $\mathrm{NO}$ availability and increased endothelium-derived vasoconstrictors $[5,6]$. This imbalance leads to impaired endothelium-dependent vasodilation, a characteristic of ED. On the other hand, ED is indicative of endothelial activation, which cultivates a pro-inflammatory, proliferative, and procoagulant setting. This setting leads to atherosclerotic plaque formation, plaque progression, and plaque complications in previously non-atherosclerotic vessels [18, 19]. Thus, the pathological activation of endothelial cells causes atherosclerotic plaque activation and the formation of vulnerable plaques. These vulnerable plaques result in plaque complications. In other words, if a patient with preexisting atheromatous plaque has normal endothelial function, the atheroma will become stable and even regress. Thus, if the endothelial function is impaired, regardless of the presence of significant lesions in the coronary arteries, the $\mathrm{CAD}$ process accelerates, and atheromatous plaques become activated. As a result, the patient symptomatically presents with severe atherosclerotic le-

Reactive Hyperemia and Coronary Artery Disease sions, as in our subjects, and without treatment develops major cardiovascular events as shown in many studies. In light of this information, endothelial function is expected to be significantly impaired in patients with major cardiovascular events. Although there are molecular studies that support this hypothesis, there are no clinical trials $[20,21]$.

Evaluation of endothelial functions with peripheral artery tonometry is a noninvasive, practical, nonoperatordependent, and safe examination method. The relationship between peripheral artery tonometry and serum NO levels has been demonstrated in several studies. Nohria et al. [22] showed that RHI decreased significantly in 23 subjects after the infusion of L-nitroarginine methyl ester (an NO synthase inhibitor). Other studies have demonstrated its correlation with invasive examination methods [7]. In a study on 150 patients with $<30 \%$ stenosis in coronary angiography, patients were divided into two groups according to the presence of impaired coronary endothelial functions as determined by intracoronary acetylcholine response. It was determined that $\mathrm{RHI}$ of $<1.35$ was a predictor of impaired intracoronary acetylcholine response [7]. The limitations of our study include the low sample size, the cross-sectional and single-center design, the evaluation with coronary angiography only atherosclerosis that extended into lumens, and not measuring biochemical markers of endothelial functions (such as ADMA).

\section{Conclusions}

This study provides diagnostic and prognostic information on the investigation of endothelial functions along with several risk factors and demonstrates that measurement of RHI could be useful in evaluating patients for CAD. It can also help optimize treatment. To the best of our knowledge, this study is the first to demonstrate the relationship between ED and the presence and extent of CAD. The measurement of RHI in low-risk to medium-risk individuals in clinical practice may help in clinical decision-making processes. Moreover, impaired endothelial function, a common indicator of cardiovascular health, can be detected in the early period even in individuals with normal coronary arteries.

\section{Statement of Ethics}

The study was approved by the local Ethics Committee and was carried out in accordance with the Declaration of Helsinki. Written informed consent was obtained from all participants. 


\section{Conflict of Interest Statement}

The authors have no conflicts of interest to declare.

\section{Author Contributions}

All authors contributed to the conception or design and the acquisition, analysis, revising, and approval of the work.

\section{Data Availability Statement}

All data generated and analyzed in this study are included in this paper. Further inquiries can be directed to the corresponding author.

\section{References}

1 Pahor M, Elam MB, Garrison RJ, Kritchevsky SB, Applegate WB. Emerging noninvasive biochemical measures to predict cardiovascular risk. Arch Intern Med. 1999 Feb 8;159(3): 237-45.

2 Widlansky ME, Gokce N, Keaney JF Jr, Vita JA. The clinical implications of endothelial dysfunction. J Am Coll Cardiol. 2003 Oct 1; 42(7):1149-60.

3 Wang J, Widlansky ME. Lifestyle choices and endothelial function: risk and relevance. Curr Vasc Pharmacol. 2009 Apr;7(2):209-24.

4 Blann AD, Lip GY. Coronary risk factors, endothelial function, and atherosclerosis: a review. Clin Cardiol. 1997 Oct;20(10):82224.

5 Ivanova OV, Soboleva GN, Karpov Iu A. Endothelial dysfunction: an important stage in the development of atherosclerotic vascular lesions (a review of the literature -1 ). Ter Arkh. 1997;69(6):75-8

6 Sharma N, Andrews TC. Endothelial function as a therapeutic target in coronary artery disease. Curr Atheroscler Rep. 2000 Jul;2(4): 303-7.

7 Bonetti PO, Pumper GM, Higano ST, Holmes DR Jr, Kuvin JT, Lerman A. Noninvasive identification of patients with early coronary atherosclerosis by assessment of digital reactive hyperemia. J Am Coll Cardiol. 2004 Dec 7;44(11):2137-41.

8 Hamburg NM, Keyes MJ, Larson MG, Vasan RS, Schnabel R, Pryde MM, et al. Cross-sectional relations of digital vascular function to cardiovascular risk factors in the Framingham Heart Study. Circulation. 2008 May 13; 117(19):2467-74.
9 Okutucu S, Jam F, Sabanoglu C, Yetis Sayin B, Aksoy H, Ercan Akgul E, et al. Fingertip reactive hyperemia peripheral arterial tonometry score predicts response to biventricular pacing. Acta Cardiol Sin. 2018 Nov;34(6):48895.

10 Kelly AE, Gensini GG. Coronary arteriography and left-heart studies. Heart Lung. 1975 Jan-Feb;4(1):85-98.

11 Deveci OS, Kabakci G, Tulumen E, Okutucu S, Aksoy H, Kaya EB, et al. The relationship between microalbuminuria and the presence and extent of coronary atherosclerosis. Angiology. 2010 Feb;61(2):184-91.

12 Ludmer PL, Selwyn AP, Shook TL, Wayne RR, Mudge GH, Alexander RW, et al. Paradoxical vasoconstriction induced by acetylcholine in atherosclerotic coronary arteries. $\mathrm{N}$ Engl J Med. 1986 Oct 23;315(17):1046-51.

13 Kuvin JT, Mammen A, Mooney P, AlsheikhAli AA, Karas RH. Assessment of peripheral vascular endothelial function in the ambulatory setting. Vasc Med. 2007 Feb;12(1):13-6.

14 SuwaidiJA, Hamasaki S, Higano ST, Nishimura RA, Holmes DR Jr, Lerman A. Long-term follow-up of patients with mild coronary artery disease and endothelial dysfunction. Circulation. 2000 Mar 7;101(9):948-54.

15 Schachinger V, Britten MB, Zeiher AM. Prognostic impact of coronary vasodilator dysfunction on adverse long-term outcome of coronary heart disease. Circulation. $2000 \mathrm{Apr}$ 25;101(16):1899-906.
16 Rubinshtein R, Kuvin JT, Soffler M, Lennon RJ, Lavi S, Nelson RE, et al. Assessment of endothelial function by non-invasive peripheral arterial tonometry predicts late cardiovascular adverse events. Eur Heart J. 2010 May; 31(9):1142-8.

17 Biasucci LM, Vitelli A, Liuzzo G, Altamura S, Caligiuri G, Monaco C, et al. Elevated levels of interleukin-6 in unstable angina. Circulation. 1996 Sep 1;94(5):874-7.

18 Kinlay S, Ganz P. Role of endothelial dysfunction in coronary artery disease and implications for therapy. Am J Cardiol. 1997 Nov 6; 80(9A):11I-6I.

19 Ross R. Atherosclerosis: an inflammatory disease. N Engl J Med. 1999 Jan 14;340(2):11526.

20 Naruse K, Shimizu K, Muramatsu M, Toki Y, Miyazaki Y, Okumura K, et al. Long-term inhibition of NO synthesis promotes atherosclerosis in the hypercholesterolemic rabbit thoracic aorta. PGH2 does not contribute to impaired endothelium-dependent relaxation. Arterioscler Thromb. 1994 May; 14(5):74652.

21 Wang BY, Singer AH, Tsao PS, Drexler H, Kosek J, Cooke JP. Dietary arginine prevents atherogenesis in the coronary artery of the hypercholesterolemic rabbit. J Am Coll Cardiol. $1994 \mathrm{Feb} ; 23(2): 452-8$.

22 Nohria A, Gerhard-Herman M, Creager MA, Hurley S, Mitra D, Ganz P. Role of nitric oxide in the regulation of digital pulse volume amplitude in humans. J Appl Physiol. 2006 Aug; 101(2):545-8. 